\title{
Evolutionary states of the two shortest period O-type overcontact binaries V382 Cyg and TU Mus
}

\author{
S.-B. Qian, ${ }^{1,2,3 \star}$ J.-Z. Yuan,,$^{1,2,3}$ L. Liu, ${ }^{1,2,3}$ J.-J. He, ${ }^{1,2,3}$ E. Fernández Lajús ${ }^{4 \star}$ \\ and J. Z. Kreiner ${ }^{5 \star}$ \\ ${ }^{1}$ National Astronomical Observatories/Yunnan Observatory, Chinese Academy of Sciences, PO Box 110, 650011 Kunming, P.R. China \\ ${ }^{2}$ United Laboratory of Optical Astronomy, Chinese Academy of Sciences (ULOAC), 100012 Beijing, P.R. China \\ ${ }^{3}$ Graduate School of the CAS, Beijing, P.R. China \\ ${ }^{4}$ Facultad de Ciencias Astronómicas y Geofísicas, Universidad Nacional de La Plata, 1900 La Plata, Buenos Aires, Argentina \\ ${ }^{5}$ Mt Suhora Observatory, Cracow Pedagogical University ul. Podchorazych 2, 30-084 Krakow, Poland
}

Accepted 2007 July 5. Received 2007 June 20; in original form 2007 April 9

\begin{abstract}
Up to now, V382 Cyg and TU Mus are the only two discovered O-type overcontact binary stars with periods less than two days $(P=1.8855$ and $1.3873 \mathrm{~d})$. Both systems contain a visual companion. New eclipse times and analyses of orbital period variations of the two systems are presented. It is discovered that the periods of both binaries show cyclic oscillations with periods of 47.70 and $47.73 \mathrm{yr}$, while they undergo continuous increases at rates of $\mathrm{d} P / \mathrm{d} t=$ $+4.4 \times 10^{-7}$ and $+4.0 \times 10^{-7} \mathrm{~d} \mathrm{yr}^{-1}$, respectively. The periodic variations can be interpreted as light travel times effects caused by the presence of invisible tertiary components suggesting that they may be quadruple systems. It is possible that the additional bodies may play an important role in the formation and evolution of the two massive overcontact binaries by removing angular momentum from the central systems, and causing the eclipsing pairs to have lower angular momentum and shorter initial orbital periods. In this way, the original detached systems can evolve into the present overcontact configurations via a Case A mass transfer. This is in agreement with the observed long-term period increase of V382 Cyg and TU Mus, which can be explained by mass transfers from the less massive components to the more massive ones. It is found that the time-scales of the long-term period variations of both systems are much longer than the thermal time-scales of the secondary components, but are close to their nuclear time-scales. This suggests that the two massive binaries have been through the rapid mass-transfer evolutionary stage on the thermal time-scales of the secondaries, and they are now on the slow phase of Case A mass transfer. It is shown that massive overcontact binaries are going through a short-lived overcontact configuration during the evolutionary phases of Case A mass transfer, which is different from the situation of late-type overcontact binary stars where components remain in good overcontact configuration driving by a combination of thermal relaxation oscillation and variable angular momentum loss via change in overcontact depth. This conclusion is in agreement with the distribution of overcontact binary stars along with the orbital period.
\end{abstract}

Key words: binaries: close - binaries: eclipsing - stars: early-type - stars: evolution - stars: individual: V382 Cyg - stars: individual: TU Mus.

\section{INTRODUCTION}

Theoretical studies have shown that massive short-period binary stars can evolve into overcontact configurations via a Case A mass

\footnotetext{
^E-mail: qsb@ynao.ac.cn (SBQ); eflajus@lilen.fcaglp.unlp.edu.ar (EFL); sfkreine@cyf-kr.edu.pl (JZK)
}

transfer (the mass transfer is taking place during the main-sequence evolutionary stage of the mass loser). Sybesma (1985) suggested that binaries with initial masses of $20+10 \mathrm{M}_{\odot}$ and initial periods shorter than $3 \mathrm{~d}$ evolve into a short-lived overcontact configuration during the rapid phase of Case A mass transfer. Subsequent investigation by Sybesma (1986) indicated that systems with higher initial mass ratios (e.g. $q=0.8$ and 0.9 ) and still shorter periods form more stable overcontact systems within the slow stage of Case A 
Table 1. Physical properties of V382 Cyg and TU Mus.

\begin{tabular}{lccccccccccc}
\hline Stars & Sp. & $P$ & $i$ & $f$ & $q$ & $M_{1}$ & $M_{2}$ & $R_{1}$ & $R_{2}$ & $\log L_{1} / \mathrm{L} \odot$ & $\log L_{2} / \mathrm{L} \odot$ \\
\hline V382 Cyg & O7.3V+O7.7V & 1.8855 & 88.2 & 22 per cent & 0.551 & 26.6 & 18.0 & 9.85 & 8.30 & 5.13 & 4.93 \\
TU Mus & O7.5V+O9.5V & 1.3873 & 77.8 & 5.0 per cent & 0.651 & 23.5 & 15.3 & 7.48 & 6.15 & 4.8 & 4.5 \\
\hline
\end{tabular}

mass transfer. By comparing observational physical parameters of seven massive overcontact binaries with stellar evolutionary grids, Hilditch \& Bell (1987) mentioned that these systems are probably in the slow phase of a Case A mass transfer. Terrell et al. (2003) pointed out that very little is known about the structure and evolution of overcontact binary systems with radiative envelope and no models of this type have been published. To our knowledge, V382 Cyg and TU Mus are the only two O-type overcontact binary stars with orbital period less than two days. Secular orbital period changes of the two binary stars are very important to understand their formation and evolution during the overcontact configuration. They constrain on the amount of mass and angular momentum transfer and also give information on the properties of evolutionary states and companion environment.

The light variability of V382 Cyg $\left(=\mathrm{HD} 228854=\mathrm{BD}+35^{\circ}\right.$ 4026) was discovered by Morgentoth (1935). Petrov (1946) later discovered that it is an eclipsing binary with a $\beta$-type light curve, which was analysed by Albo (1958). Pearce (1952) classified the spectral types of both components as O6.5+O7.5, and derived the masses of 37.3 and $26.3 \mathrm{M}_{\odot}$ by combining his spectroscopic observations with the photometric data of Petrov (1946). These properties make the system one of the most massive binary stars known. Photoelectric light curves in $U B V$ bands were published by Landolt $(1964,1975)$ and photometric solutions were derived by Landolt (1964) with the Russell-Merrill method, by Cester et al. (1978) with Wood's WINK method and by Devinney \& Twigg (1974) with the Wilson-Devinney method. The photometric solutions of Cester et al. (1978) suggest that the system is an overcontact binary with masses of 38 and $33.3 \mathrm{M}_{\odot}$ for the primary and the secondary component. The IUE spectra of V382 Cyg were obtained by Koch, Siah $\&$ Fanelli (1979). Popper (1980) listed the spectral types of the components as 07.3+O7.7. The spectroscopic observations of Popper (1978) were analysed by Popper \& Hill (1991) who derived the masses of both components as 32.6 and $22.9 \mathrm{M}_{\odot}$. Harries, Hilditch $\&$ Hill (1997) subsequently derived spectroscopic and photometric solutions of the binary stars by using the LIGHT 2 method. They obtained the masses of 26 and $19.3 \mathrm{M}_{\odot}$ that are significantly lower than the previous ones. More recently, Değirmenci et al. (1999) published new photometric light curves and photometric solutions. They showed that V382 is a massive overcontact binary system with a degree of overcontact of 22 per cent.

The light variability of the other short-period O-type binary star, TU Mus (=HD 100213), was discovered by Oosterhoff (1928). Both photometric and spectroscopic observations of TU Mus were obtained by Anderson \& Grønbech (1975) who estimated the spectral type of the primary as 07-O8 and derived the masses of both components as 23.5 and $15.8 \mathrm{M}_{\odot}$. The light curves of Anderson \& Grønbech (1975) were later analysed by Wilson \& Rafert (1981). Radial velocity curves of the two components of TU Mus based on IUE spectra were obtained by Stickland et al. (1995) who determined $M_{1} \sin ^{3} i=15.70 \mathrm{M}_{\odot}$ and $M_{2} \sin ^{3} i=9.85 \mathrm{M}_{\odot}$. These values are much lower than those derived by Anderson \& Grønbech (1975). Terrell et al. (2003) recently published new photometric and spectroscopic observations and a detailed analysis of those data. They classified the spectral types of both components as O7.5+09.5. Their solutions showed that TU Mus is an overcontact binary with a small overcontact degree of 5 per cent. The absolute parameters they derived are $M_{1}=23.5$ and $M_{2}=15.3 \mathrm{M}_{\odot}$, which are in good agreement with those obtained by Anderson \& Grønbech (1975).

The physical parameters of V382 Cyg and TU Mus obtained by Değirmenci et al. (1999) and Terrell et al. (2003) are displayed in Table 1. As shown in the table, physical properties of the two eclipsing binaries are nearly the same. Moreover, both systems contain a visual companion (see e.g. Chambliss 1992; Terrell et al. 2003). In the present paper, the changes of their orbital periods were investigated. Then, based on the period variations, the multiplicity, the formation and the evolutionary states of the two massive binaries are compared with those of late-type overcontact binary stars.

\section{ORBITAL PERIOD VARIATIONS OF V382 CYG}

Epochs and orbital periods of V382 Cyg have been given by several authors (e.g. Landolt 1975; Mayer et al. 1998) and are listed in Table 2. As shown in the table, the period of the binary star derived recently by Mayer et al. (1998) and Değirmenci et al. (1999) is longer than those derived by former authors indicating that the period of V382 is increasing. Because the derived period by Landolt (1975) is identical to the period given by Whitney (1959) (see Table 2), Landolt concluded that the period of the massive binary is constant. Three abrupt variations in the period of V382 Cyg were reported by Koch et al. (1979), while Mayer (1980) argued that these changes are not real and derived a quadratic ephemeris,

$$
\begin{aligned}
\text { Min.I }= & 2436814.7658+1.8855148 d \times E \\
& +0.98 \times 10^{-9} \times E^{2},
\end{aligned}
$$

which corresponds to a period increase of $\mathrm{d} P / \mathrm{d} t=+3.8 \times$ $10^{-7} \mathrm{~d} \mathrm{yr}^{-1}$. However, the ephemeris was supported only by two earlier photographic minima. If the two minima were removed, a linear ephemeris also can fit the rest data well. Therefore, based on only 12 photoelectric times of light minimum, Mayer et al. (1986) showed that the period undergoes a cyclic change with a period of $7.1 \mathrm{yr}$ and an amplitude of $0.0050 \mathrm{~d}$. A more recent period analysis by Mayer et al. (1998) indicates a sudden period increase (1.6 s) around

Table 2. Epochs and orbital periods of V382 Cyg.

\begin{tabular}{lll}
\hline Epochs & Orbital period & References \\
\hline 2433539.3710 & 1.885497 & Pearce (1952) \\
2427992.458 & 1.885512 & Whitney (1959) \\
2432172.644 & 1.885504 & Landolt (1964) \\
2436814.7735 & 1.8855121 & Landolt (1975) \\
2436814.655 & 1.8855121 & Koch et al. (1979) \\
2436814.7706 & 1.8855146 & Mayer (1980) \\
2436814.7703 & 1.8855160 & Mayer et al. (1986) \\
2436814.680 & 1.8855328 & Mayer et al. (1998) \\
2436814.6751 & 1.88553364 & Değirmenci et al. (1999) \\
\hline
\end{tabular}


Table 3. New CCD times of light minimum of V382 Cyg and TU Mus.

\begin{tabular}{lllll}
\hline Stars & JD (Hel.) (d) & Error (d) & Min. & Filter \\
\hline V382 Cyg & 2453837.2977 & \pm 0.0005 & I & $R$ \\
TU Mus & 2450951.645 & \pm 0.001 & I & No \\
TU Mus & 2450983.552 & \pm 0.001 & I & $V$ \\
\hline
\end{tabular}

HJD 244 5766, whereas the period study by Deǧirmenci et al. (1999) suggests a continuous period increase. They derived the following ephemeris:

$$
\begin{aligned}
\text { Min.I }= & 2436814.7642+1.8855132 d \times E \\
& +0.98 \times 10^{-9} \times E^{2},
\end{aligned}
$$

where the quadratic term is the same as that determined by Mayer (1980).

The fact that different patterns of period change, e.g. continuous period increase, small-amplitude oscillation and sudden period increase, were reported for V382 Cyg suggests that the period change of the binary star is complex. In order to study the period change of the binary system, we have monitored it on 2006 April 11 with the PI1024 TKB CCD photometric system attached to the $1.0-\mathrm{m}$ reflecting telescope at the Yunnan Observatory in China. During the observation, the integration time for each image was $50 \mathrm{~s}$ and the $R_{\mathrm{J}}$ band we used are close to that in the standard Johnson UBVRI system. The obtained images were reduced with PHOT (measure magnitudes for a list of stars) of the aperture photometry package of IRAF. By using a parabolic fitting method, we determined one epoch of minimum light which is listed in Table 3. All available times of light minimum were collected in 2006 by one of us (Kreiner, private communication). The $(O-C)_{1}$ residuals of those data with respect to the ephemeris given by Kreiner, Kim \& Nha (2001),

Min..$=2436814.802+1.8855109 d \times E$,

were computed and are shown in Table 4. The corresponding $(O-C)_{1}$ diagram is displayed in the upper panel of Fig. 1 where open circles refer to photographic or visual data and solid dots to photoelectric and CCD observations.

As shown in Fig. 1, the general trend of the $(O-C)_{1}$ curve shows an upward parabolic change, indicating that the period is increasing continuously, as has been reported by Mayer (1980) and Değirmenci et al. (1999). However, after the long-term continuous increase was removed from the $(O-C)_{1}$ curve, we found there is a cyclic oscillation in the residuals. Therefore, a cyclic term is added to a quadratic ephemeris to give a good fit to the observations (solid line in Fig. 1). By considering that the error of visual and photographic (VP) observations is about $0.008 \mathrm{~d}$ and that of photoelectric and CCD (PC) data is about $0.001 \mathrm{~d}$, we choose weights 1 for VP data and 8 for PC observations. A weighted least-squares solution leads to the following quadratic ephemeris:

$$
\begin{aligned}
\text { Min.I }= & 2436814.7610( \pm 0.0021) \\
& +1.88550741 d( \pm 0.00000027) \times E \\
& +1.13( \pm 0.05) \times 10^{-9} \times E^{2} \\
& +0.0148( \pm 0.0020) \sin [0.0390 \times E+71.08( \pm 0.06)] .
\end{aligned}
$$

The quadratic term in the ephemeris (dashed line in Fig. 1) suggests a continuous period increase at a rate of $\mathrm{d} P / \mathrm{d} t=+4.4( \pm 0.2) \times$
$10^{-7} \mathrm{~d} \mathrm{yr}^{-1}$, which corresponds to a period increase of $3.8 \mathrm{~s}$ per century. The present period increase rate is close to that derived by Mayer (1980) and Değirmenci et al. (1999). The residuals from the equation are displayed in the low panel of Fig. 1.

The sinusoidal term in equation (4) reveals a periodic change with a period of $P_{3}=47.7 \mathrm{yr}$ and an amplitude of $A_{3}=0.0148 \mathrm{~d}$, which is more easily seen in Fig. 2 where the $(\mathrm{O}-\mathrm{C})_{2}$ values calculated from the quadratic part of equation (4) are plotted and the solid line in the figure represents the fit by the periodic term in equation (4).

\section{ORBITAL PERIOD CHANGES OF TU MUS}

Linear ephemerides of TU Mus have been determined by several authors (e.g. Anderson \& Grønbech 1975). Terrell et al. (2003) derived the following linear ephemeris:

Min. $\mathrm{I}=2448500.3080+1.38728653 d \times E$,

and pointed out that the period of TU Mus has remained constant over 30 years. However, they did not include the old times of light minimum determined by Oosterhoff $(1928,1930)$ and by Knipe (1971). Recently, Mayer (2004) determined one eclipsing time by using the All Sky Automated Survey (ASAS) data base (Pojmanski 2002) and pointed out that the period of the binary is increasing as in the case of V382 Cyg. After the work of Mayer (2004), several unpublished times were determined by Zakrzewski and Ogloza (private communication). All of the available data have been collected in 2006 by one of us (Kreiner, private communication).

Two times of light minimum of TU Mus were obtained by one of the co-authors (Dr Fernández Lajús) several years ago and are listed in Table 3. These observations of TU Mus were obtained with the 0.8-m Cassegrain reflector telescope $(\mathrm{f} / 20.06)$ at the La Plata observatory in Argentina. The field of view of the CCD photometric system is $1 \operatorname{arcmin} 54 \operatorname{arcsec} \times 2 \operatorname{arcmin} 50 \operatorname{arcsec}$. The $(O-C)_{1}$ curve of TU Mus was formed by the linear ephemeris of Kreiner et al. (2001),

Min.I $=2441669.3255+1.3872766 d \times E$,

and is displayed in Fig. 3 where open circles refer to photographic or visual observations and solid dots to photoelectric and CCD data. The corresponding $(O-C)_{1}$ values are listed in Table 5. As shown in Fig. 3, the period changes of TU Mus appear to resemble those of V382 Cyg. Therefore, a sinusoidal term was added to a quadratic ephemeris to get a good fit to the observations (solid line in Fig. 3). With the same weights as those used for V382 Cyg, a weighted least-square solution yields the following ephemeris:

$$
\begin{aligned}
\text { Min.I }= & 2441669.3099( \pm 0.0073) \\
& +1.38727095 d( \pm 0.00000113) \times E \\
& +5.96( \pm 0.93) \times 10^{-10} \times E^{2} \\
& +0.0100( \pm 0.0054) \sin [0.0286 \times E+346.1( \pm 9.9)] .
\end{aligned}
$$

The residuals from this equation are displayed in the lower panel of Fig. 3. The quadratic term in equation (7) indicates a long-term period increase at the rate of $\mathrm{d} P / \mathrm{d} t=+4.0( \pm 0.5) \times 10^{-7} \mathrm{~d} \mathrm{yr}^{-1}$, which corresponds to a period increase of $3.46 \mathrm{~s}$ per century.

The $(\mathrm{O}-\mathrm{C})_{2}$ residuals with respect to the quadratic ephemeris in equation (7) are shown in Fig. 4 and are listed in Column 6 of Table 5. As displayed in Fig. 4, a cyclic oscillation is clearly seen. The sinusoidal term in equation (7) indicates a small-amplitude 
Table 4. Times of light minimum of V382 Cyg.

\begin{tabular}{|c|c|c|c|c|c|c|c|}
\hline $\begin{array}{l}\text { JD (Hel.) } \\
2400000+\end{array}$ & $\begin{array}{l}\text { Errors } \\
\text { (d) }\end{array}$ & Min. & Method & E & $\begin{array}{l}(O-C)_{1} \\
\text { (d) }\end{array}$ & $\begin{array}{l}(\mathrm{O}-\mathrm{C})_{2} \\
\text { (d) }\end{array}$ & $\begin{array}{l}\text { Residuals } \\
\text { (d) }\end{array}$ \\
\hline 13488.26 & & II & $\mathrm{Pg}$ & -12371.5 & +0.0561 & -0.0332 & -0.0218 \\
\hline 15165.44 & & I & $\mathrm{Pg}$ & -11482 & +0.0742 & +0.0058 & +0.0099 \\
\hline 27991.488 & & II & Vis & -4679.5 & -0.0657 & -0.0332 & -0.0194 \\
\hline 27992.436 & & I & Vis & -4679 & -0.0605 & -0.0332 & -0.0194 \\
\hline 27992.442 & & I & Vis & -4679 & -0.0565 & -0.0240 & -0.0102 \\
\hline 28108.406 & \pm 0.0080 & II & Vis & -4617.5 & -0.0494 & +0.0236 & +0.0376 \\
\hline 28145.183 & \pm 0.0038 & I & Vis & -4598 & -0.0399 & -0.0068 & +0.0073 \\
\hline 28431.743 & & I & $\mathrm{Pg}$ & -4446 & -0.0775 & -0.0434 & -0.0289 \\
\hline 28746.65 & & I & $\mathrm{Pg}$ & -4279 & -0.0509 & -0.0157 & -0.0010 \\
\hline 29014.41 & & I & $\mathrm{Pg}$ & -4137 & -0.0334 & +0.0027 & +0.0175 \\
\hline 29457.52 & & I & $\mathrm{Pg}$ & -3902 & -0.0185 & +0.0189 & +0.0335 \\
\hline 32172.607 & & I & $\mathrm{Pg}$ & -2462 & -0.0672 & -0.0245 & -0.0183 \\
\hline 32386.651 & & II & $\mathrm{Pg}$ & -2348.5 & -0.0287 & +0.0143 & +0.0194 \\
\hline 32798.621 & & I & $\mathrm{Pg}$ & -2130 & -0.0428 & +0.0005 & +0.0035 \\
\hline 33061.660 & & II & $\mathrm{Pg}$ & -1990.5 & -0.0326 & +0.0109 & +0.0125 \\
\hline 33111.623 & & I & $\mathrm{Pg}$ & -1964 & -0.0356 & +0.0079 & +0.0090 \\
\hline 33503.800 & & I & $\mathrm{Pg}$ & -1756 & -0.0449 & -0.0013 & -0.0020 \\
\hline 33567.935 & & I & $\mathrm{Pg}$ & -1722 & -0.0172 & +0.0265 & +0.0254 \\
\hline 33834.713 & & II & $\mathrm{Pg}$ & -1580.5 & -0.0390 & +0.0047 & +0.0022 \\
\hline 33886.563 & & I & $\mathrm{Pg}$ & -1553 & -0.0406 & +0.0031 & +0.0003 \\
\hline 34182.594 & & I & $\mathrm{Pg}$ & -1396 & -0.0348 & +0.0089 & +0.0046 \\
\hline 34296.666 & & II & $\mathrm{Pg}$ & -1335.5 & -0.0362 & +0.0074 & +0.0026 \\
\hline 35019.773 & & I & $\mathrm{Pg}$ & -952 & -0.0226 & +0.0207 & +0.0124 \\
\hline 35337.467 & & II & $\mathrm{Pg}$ & -783.5 & -0.0392 & +0.0038 & -0.0058 \\
\hline 35483.605 & & I & $\mathrm{Pg}$ & -706 & -0.0263 & +0.0166 & +0.0064 \\
\hline 36814.7725 & \pm 0.0010 & I & $\mathrm{Pe}$ & 0 & -0.0295 & +0.0115 & -0.0025 \\
\hline 36837.392 & & I & $\mathrm{Pg}$ & 12 & -0.0361 & +0.0049 & -0.0092 \\
\hline 37940.428 & & I & $\mathrm{Pg}$ & 579 & -0.0240 & +0.0145 & -0.0002 \\
\hline 37959.321 & & I & $\mathrm{Pg}$ & 607 & 0.0139 & +0.0524 & +0.0376 \\
\hline 38987.8298 & \pm 0.0010 & II & $\mathrm{Pe}$ & 1152.5 & -0.0235 & +0.0120 & -0.0013 \\
\hline 40385.931 & \pm 0.0030 & I & $\mathrm{Pe}$ & 1894 & -0.0286 & +0.0017 & -0.0068 \\
\hline 40386.8761 & \pm 0.0004 & II & $\mathrm{Pe}$ & 1894.5 & -0.0263 & +0.0041 & -0.0045 \\
\hline 40387.8173 & \pm 0.0011 & I & $\mathrm{Pe}$ & 1895 & -0.0279 & +0.0024 & -0.0061 \\
\hline 41129.7698 & \pm 0.0002 & II & $\mathrm{Pe}$ & 2288.5 & -0.0239 & +0.0032 & -0.0018 \\
\hline 42651.3844 & \pm 0.0014 & II & $\mathrm{Pe}$ & 3095.5 & -0.0166 & +0.0027 & +0.0058 \\
\hline 42659.8678 & & I & $\mathrm{Pe}$ & 3100 & -0.0180 & +0.0013 & +0.0044 \\
\hline 42940.8071 & \pm 0.0002 & I & $\mathrm{Pe}$ & 3249 & -0.0198 & -0.0021 & +0.0024 \\
\hline 42961.5424 & & I & $\mathrm{Pe}$ & 3260 & -0.0251 & -0.0075 & -0.0029 \\
\hline 43366.9333 & & I & $\mathrm{Pe}$ & 3475 & -0.0191 & -0.0039 & +0.0027 \\
\hline 44445.4450 & & I & $\mathrm{Pe}$ & 4047 & -0.0196 & -0.0113 & -0.0001 \\
\hline 44757.493 & & II & $\mathrm{Pe}$ & 4212.5 & -0.0237 & -0.0175 & -0.0053 \\
\hline 44842.3538 & & II & $\mathrm{Pe}$ & 4257.5 & -0.0109 & -0.0053 & +0.0071 \\
\hline 45598.4426 & \pm 0.0007 & II & $\mathrm{Pe}$ & 4658.5 & -0.0119 & -0.0118 & +0.0024 \\
\hline 46274.3971 & \pm 0.0020 & I & $\mathrm{Pe}$ & 5017 & -0.0131 & -0.0182 & -0.0034 \\
\hline 46325.3087 & \pm 0.0009 & I & $\mathrm{Pe}$ & 5044 & -0.0103 & -0.0158 & -0.0010 \\
\hline 46668.4782 & \pm 0.0006 & I & $\mathrm{Pe}$ & 5226 & -0.0038 & -0.0120 & +0.0027 \\
\hline 47030.4992 & & I & $\mathrm{Pe}$ & 5418 & -0.0079 & -0.0191 & -0.0046 \\
\hline 47099.3141 & & II & $\mathrm{Pe}$ & 5454.5 & -0.0071 & -0.0189 & -0.0045 \\
\hline 47362.370 & & I & Vis & 5594 & 0.0200 & +0.0060 & +0.0200 \\
\hline 47426.462 & & I & Vis & 5628 & 0.0047 & -0.0099 & +0.0040 \\
\hline 47444.3744 & & II & $\mathrm{Pe}$ & 5637.5 & 0.0047 & -0.0100 & +0.0038 \\
\hline 47461.327 & & II & Vis & 5646.5 & -0.0123 & -0.0272 & -0.0134 \\
\hline 47757.392 & & II & Vis & 5803.5 & 0.0275 & +0.0100 & +0.0232 \\
\hline 47823.366 & & II & Vis & 5838.5 & 0.0086 & -0.0094 & +0.0035 \\
\hline 48069.420 & & I & Vis & 5969 & +0.0034 & -0.0168 & -0.0045 \\
\hline 48085.450 & & II & Vis & 5977.5 & +0.0066 & -0.0138 & -0.0015 \\
\hline 48167.4747 & & I & $\mathrm{Pe}$ & 6021 & 0.0116 & -0.0095 & +0.0025 \\
\hline 48167.4765 & & I & $\mathrm{Pe}$ & 6021 & 0.0134 & -0.0077 & +0.0043 \\
\hline 48186.3306 & & I & $\mathrm{Pe}$ & 6031 & 0.0124 & -0.0089 & +0.0030 \\
\hline 48186.3316 & & I & $\mathrm{Pe}$ & 6031 & 0.0134 & -0.0079 & +0.0040 \\
\hline 48447.4743 & \pm 0.0004 & II & $\mathrm{Pe}$ & 6169.5 & 0.0128 & -0.0109 & +0.0002 \\
\hline 48447.4746 & \pm 0.0002 & II & $\mathrm{Pe}$ & 6169.5 & 0.0149 & -0.0088 & +0.0023 \\
\hline 48498.3835 & \pm 0.0017 & II & $\mathrm{Pe}$ & 6169.5 & 0.0132 & -0.0105 & +0.0006 \\
\hline
\end{tabular}


Table 4 - continued

\begin{tabular}{|c|c|c|c|c|c|c|c|}
\hline $\begin{array}{l}\text { JD (Hel.) } \\
2400000+\end{array}$ & $\begin{array}{l}\text { Errors } \\
\text { (d) }\end{array}$ & Min. & Method & $\begin{array}{l}E \\
\text { (d) }\end{array}$ & $\begin{array}{l}(O-C)_{1} \\
\text { (d) }\end{array}$ & $\begin{array}{l}(O-C)_{2} \\
\text { (d) }\end{array}$ & Residuals \\
\hline 48498.3844 & \pm 0.0006 & II & $\mathrm{Pe}$ & 6169.5 & 0.0141 & -0.0096 & +0.0015 \\
\hline 48564.374 & \pm 0.0060 & II & $\mathrm{Pe}$ & 6231.5 & 0.0108 & -0.0140 & -0.0034 \\
\hline 48839.6629 & \pm 0.0003 & II & $\mathrm{Pe}$ & 6377.5 & 0.0151 & -0.0123 & -0.0027 \\
\hline 48843.4381 & \pm 0.0030 & II & $\mathrm{Pe}$ & 6379.5 & 0.0193 & -0.0081 & +0.0014 \\
\hline 48843.4395 & \pm 0.0005 & II & $\mathrm{Pe}$ & 6379.5 & 0.0207 & -0.0067 & +0.0028 \\
\hline 48843.4397 & \pm 0.0005 & II & $\mathrm{Pe}$ & 6379.5 & 0.0209 & -0.0065 & +0.0030 \\
\hline 48859.4664 & \pm 0.0021 & I & $\mathrm{Pe}$ & 6388 & 0.0208 & -0.0068 & +0.0027 \\
\hline 48860.382 & \pm 0.0060 & II & Vis & 6388.5 & -0.0064 & -0.0340 & -0.0245 \\
\hline 48878.3252 & \pm 0.0005 & I & $\mathrm{Pe}$ & 6398 & 0.0245 & -0.0033 & +0.0062 \\
\hline 49221.4844 & \pm 0.0018 & I & $\mathrm{Pe}$ & 6580 & 0.0207 & -0.0104 & -0.0024 \\
\hline 49221.4857 & \pm 0.0016 & I & $\mathrm{Pe}$ & 6580 & 0.0220 & -0.0091 & -0.0011 \\
\hline 49518.4527 & \pm 0.0013 & II & $\mathrm{Pe}$ & 6737.5 & 0.0210 & -0.0130 & -0.0065 \\
\hline 49534.4780 & \pm 0.0006 & I & $\mathrm{Pe}$ & 6746 & 0.0195 & -0.0147 & -0.0082 \\
\hline 49550.5029 & \pm 0.0035 & II & $\mathrm{Pe}$ & 6754.5 & 0.0175 & -0.0168 & -0.0102 \\
\hline 49567.4771 & \pm 0.0009 & II & $\mathrm{Pe}$ & 6763.5 & 0.0221 & -0.0124 & -0.0061 \\
\hline 49568.4222 & \pm 0.0013 & I & $\mathrm{Pe}$ & 6764 & 0.0245 & -0.0100 & -0.0037 \\
\hline 49880.4846 & & II & $\mathrm{Pe}$ & 6929.5 & 0.0348 & -0.0028 & +0.0019 \\
\hline 49913.4766 & & I & $\mathrm{Pe}$ & 6947 & 0.0304 & -0.0076 & -0.0030 \\
\hline 49913.4785 & \pm 0.0010 & I & $\mathrm{Pe}$ & 6947 & +0.0323 & -0.0057 & -0.0011 \\
\hline 49915.3654 & & I & $\mathrm{Pe}$ & 6948 & +0.0337 & -0.0043 & +0.0003 \\
\hline 49929.5074 & & II & $\mathrm{Pe}$ & 6955.5 & 0.0343 & -0.0038 & +0.0007 \\
\hline 49930.4476 & & I & $\mathrm{Pe}$ & 6956 & 0.0318 & -0.0064 & -0.0018 \\
\hline 49931.3909 & & II & $\mathrm{Pe}$ & 6956.5 & 0.0323 & -0.0059 & -0.0014 \\
\hline 49945.5342 & & I & $\mathrm{Pe}$ & 6964 & 0.0343 & -0.0041 & +0.0004 \\
\hline 49946.4757 & & II & $\mathrm{Pe}$ & 6964.5 & 0.0330 & -0.0053 & -0.0010 \\
\hline 49947.4157 & \pm 0.0009 & I & $\mathrm{Pe}$ & 6965 & 0.0303 & -0.0080 & -0.0036 \\
\hline 49947.4171 & & I & $\mathrm{Pe}$ & 6965 & 0.0317 & -0.0066 & -0.0022 \\
\hline 50671.4673 & \pm 0.0004 & I & $\mathrm{Pe}$ & 7349 & 0.0457 & -0.0002 & +0.0004 \\
\hline 51413.4316 & \pm 0.0011 & II & $\mathrm{Pe}$ & 7742.5 & 0.0615 & +0.0075 & +0.0042 \\
\hline 51429.4568 & \pm 0.0007 & I & $\mathrm{Pe}$ & 7751 & 0.0598 & +0.0056 & +0.0022 \\
\hline 52532.511 & \pm 0.023 & I & Vis & 8336 & 0.0901 & +0.0232 & +0.0145 \\
\hline 52533.479 & \pm 0.017 & II & Vis & 8336.5 & 0.1154 & +0.0485 & +0.0398 \\
\hline 52817.2130 & & I & $\mathrm{CCD}$ & 8487 & 0.0800 & +0.0097 & -0.0002 \\
\hline 53837.2977 & \pm 0.0005 & I & CCD & 9028 & 0.1033 & +0.0204 & +0.0072 \\
\hline
\end{tabular}
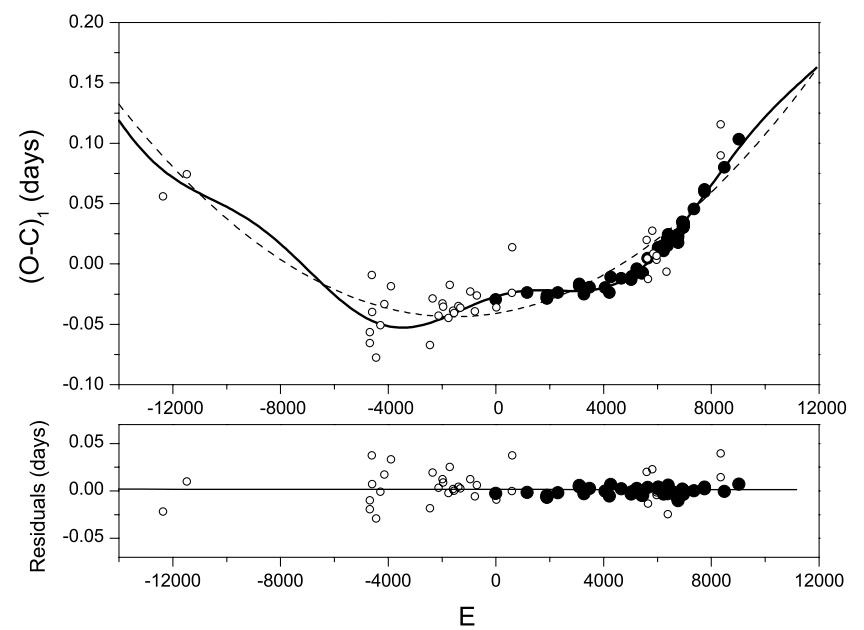

Figure 1. The $(O-C)_{1}$ curve of $\mathrm{V} 382 \mathrm{Cyg}$ computed with the linear ephemeris of Kreiner et al. (2001). The solid line refers to a combination of a continual period increase and a cyclic period variation, while the dashed line represents the long-term period increase. The residuals from equation (4) are displayed in the low panel. Open circles refer to visual or photographic data and solid circles to photoelectric and CCD observations.

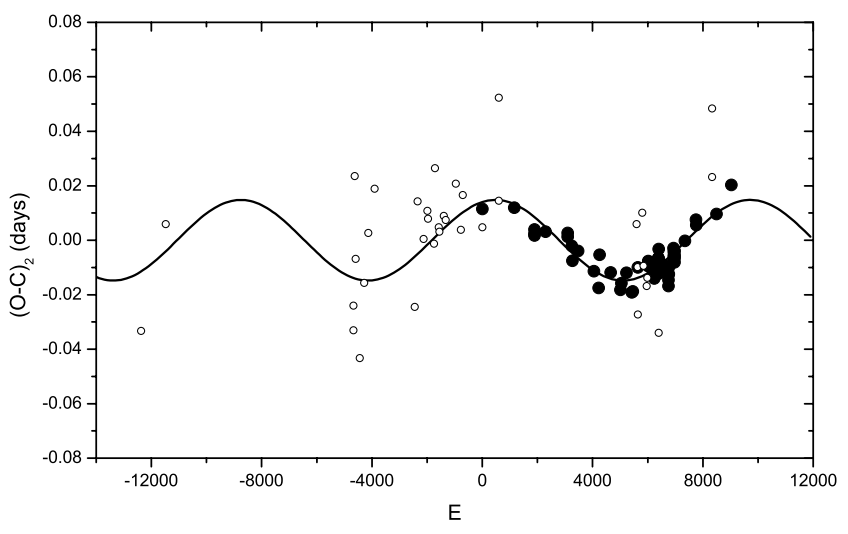

Figure 2. The $(\mathrm{O}-\mathrm{C})_{2}$ residuals for $\mathrm{V} 382 \mathrm{Cyg}$ after the continuous increase was removed from the whole period change. The solid line refers to the cyclic period oscillation. Symbols are the same as those in Fig. 1.

periodic change with a period of $P_{3}=47.73 \mathrm{yr}$ and an amplitude of $A_{3}=0.0100 \mathrm{~d}$.

\section{MECHANISMS FOR THE PERIOD CHANGES}

Based on the analyses of the O-C curves of two shortest-period O-type contact binaries V382 Cyg and TU Mus, it appears that 


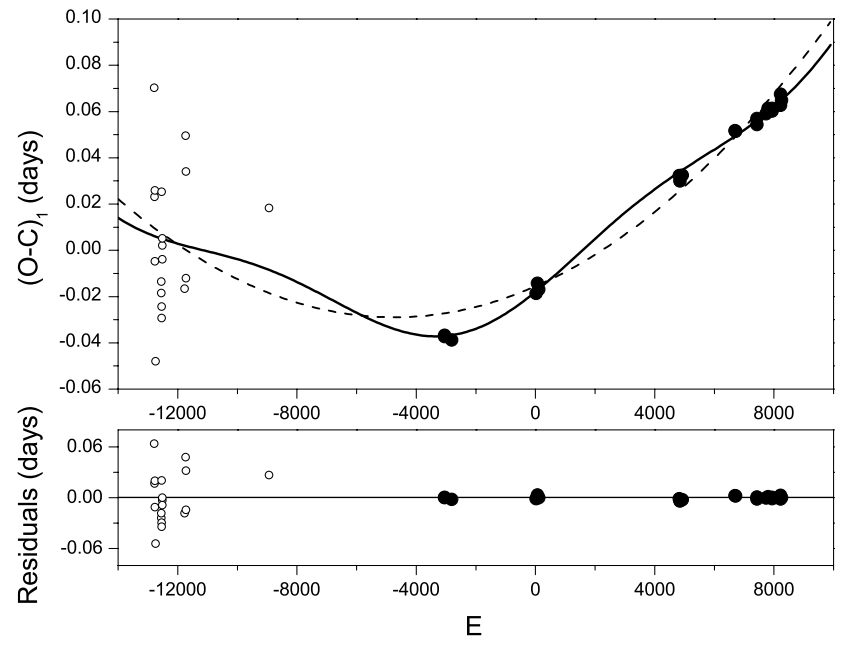

Figure 3. The same as Fig. 1, but for TU Mus. sinusoidal oscillations are discovered to be superimposed on secular period increases. In this section, mechanisms that may cause the period variations are discussed.

\subsection{Mass transfer between the components}

The general trends of the $(O-C)_{1}$ diagrams in Figs 1 and 3 (dashed line) reveal long-term period increases at rates of $\mathrm{d} P / \mathrm{d} t=+4.4 \times$ $10^{-7}$ and $+4.0 \times 10^{-7} \mathrm{~d} \mathrm{yr}^{-1}$ for V382 Cyg and TU Mus, respectively. Photometric investigations of the two binary stars indicate that they are overcontact systems with degrees of overcontact of 22 and 5.8 per cent. The secular period increases may be caused by mass transfers from the less massive components to the more massive ones. The time-scales of the period changes of the two binaries are calculated to be $\tau_{P}=P / \dot{P}=4.3 \times 10^{6}$ and $3.5 \times 10^{6} \mathrm{yr}$. The thermal and nuclear time-scales of the secondary component stars of both systems are as follows:

$\tau_{\mathrm{Th}}=2 \times 10^{7} M_{2}^{2} / L_{2} R_{2}$

and

$\tau_{\mathrm{N}}=10^{10} M_{2} / L_{2}$,

Table 5. Times of light minimum of TU Mus.

\begin{tabular}{|c|c|c|c|c|c|c|c|}
\hline $\begin{array}{l}\text { JD (Hel.) } \\
2400000+\end{array}$ & $\begin{array}{l}\text { Errors } \\
\text { (d) }\end{array}$ & Min. & Method & $E$ & $\begin{array}{l}(O-C)_{1} \\
\text { (d) }\end{array}$ & $\begin{array}{l}(\mathrm{O}-\mathrm{C})_{2} \\
\text { (d) }\end{array}$ & $\begin{array}{l}\text { Residuals } \\
\text { (d) }\end{array}$ \\
\hline 23916.417 & & I & $\mathrm{Pg}$ & -12797 & +0.0702 & +0.0605 & +0.0640 \\
\hline 23941.341 & & I & $\mathrm{Pg}$ & -12779 & +0.0232 & +0.0136 & +0.0170 \\
\hline 23966.284 & & I & $\mathrm{Pg}$ & -12761 & -0.0048 & -0.0142 & -0.0109 \\
\hline 23973.251 & & I & $\mathrm{Pg}$ & -12756 & +0.0258 & +0.0165 & +0.0198 \\
\hline 23991.212 & & I & $\mathrm{Pg}$ & -12743 & -0.0478 & -0.0570 & -0.0538 \\
\hline 24260.373 & & I & $\mathrm{Pg}$ & -12549 & -0.0184 & -0.0258 & -0.0235 \\
\hline 24260.378 & & I & $\mathrm{Pg}$ & -12549 & -0.0134 & -0.0208 & -0.0185 \\
\hline 24281.226 & & I & $\mathrm{Pg}$ & -12534 & +0.0254 & +0.0181 & +0.0204 \\
\hline 24285.338 & & I & $\mathrm{Pg}$ & -12531 & -0.0244 & -0.0316 & -0.0294 \\
\hline 24287.414 & & II & $\mathrm{Pg}$ & -12529.5 & -0.0293 & -0.0365 & -0.0343 \\
\hline 24290.220 & & II & $\mathrm{Pg}$ & -12527.5 & +0.0021 & -0.0051 & -0.0029 \\
\hline 24292.295 & & I & $\mathrm{Pg}$ & -12526 & -0.0038 & -0.0110 & -0.0088 \\
\hline 24294.385 & & II & $\mathrm{Pg}$ & -12524.5 & +0.0053 & -0.0019 & +0.0003 \\
\hline 25351.468 & & II & $\mathrm{Pg}$ & -11762.5 & -0.0165 & -0.0169 & -0.0185 \\
\hline 25388.297 & & I & $\mathrm{Pg}$ & -11736 & +0.0497 & +0.0495 & +0.0478 \\
\hline 25404.235 & & II & $\mathrm{Pg}$ & -11724.5 & +0.0340 & +0.0339 & +0.0321 \\
\hline 25417.368 & & I & $\mathrm{Pg}$ & -11715 & -0.0121 & -0.0121 & -0.0140 \\
\hline 29261.542 & & I & $\mathrm{Pg}$ & -8944 & +0.0184 & +0.0368 & +0.0268 \\
\hline 37437.401 & \pm 0.001 & II & $\mathrm{Pe}$ & -3050.5 & -0.0372 & -0.0099 & -0.0001 \\
\hline 37442.257 & \pm 0.001 & I & $\mathrm{Pe}$ & -3047 & -0.0367 & -0.0094 & +0.0004 \\
\hline 37768.265 & \pm 0.001 & I & $\mathrm{Pe}$ & -2812 & -0.0387 & -0.0119 & -0.0020 \\
\hline 41699.8270 & \pm 0.0002 & I & $\mathrm{Pe}$ & 22 & -0.0186 & -0.0031 & -0.0009 \\
\hline 41751.8543 & \pm 0.0002 & II & $\mathrm{Pe}$ & 59.5 & -0.0142 & +0.0010 & +0.0031 \\
\hline 41851.6664 & \pm 0.0003 & II & $\mathrm{Pe}$ & 105.5 & -0.0168 & -0.0018 & +0.0001 \\
\hline 48352.5629 & \pm 0.0020 & II & CCD & 4817.5 & +0.0324 & +0.0069 & -0.0013 \\
\hline 48390.7106 & \pm 0.0008 & I & CCD & 4845 & +0.0300 & +0.0042 & -0.0040 \\
\hline 48500.3080 & & I & CCD & 4924 & +0.0325 & +0.0058 & -0.0022 \\
\hline 50951.645 & \pm 0.001 & I & $\mathrm{CCD}$ & 6691 & +0.0518 & +0.0029 & +0.0025 \\
\hline 50983.552 & \pm 0.001 & I & $\mathrm{CCD}$ & 6714 & +0.0514 & +0.0022 & +0.0019 \\
\hline 51971.9895 & \pm 0.0019 & II & $\mathrm{CCD}$ & 7426.5 & +0.0543 & -0.0050 & -0.0017 \\
\hline 51974.0731 & \pm 0.0010 & I & $\mathrm{CCD}$ & 7428 & +0.0570 & -0.0023 & +0.0009 \\
\hline 52402.7436 & & I & CCD & 7737 & +0.0590 & -0.0048 & -0.0002 \\
\hline 52492.9188 & & I & $\mathrm{CCD}$ & 7802 & +0.0613 & -0.0035 & +0.0014 \\
\hline 52675.3459 & \pm 0.0010 & II & CCD & 7933.5 & +0.0615 & -0.0053 & +0.0002 \\
\hline 52680.2001 & \pm 0.0013 & I & CCD & 7937 & +0.0602 & -0.0066 & -0.0011 \\
\hline 53061.7036 & \pm 0.0008 & I & $\mathrm{CCD}$ & 8212 & +0.0627 & -0.0083 & -0.0017 \\
\hline 53066.5639 & \pm 0.0010 & II & $\mathrm{CCD}$ & 8215.5 & +0.0675 & -0.0036 & +0.0030 \\
\hline 53119.2779 & \pm 0.0002 & II & $\mathrm{Pe}$ & 8253.5 & +0.0650 & -0.0067 & +0.0001 \\
\hline
\end{tabular}


Table 6. Time-scales of V382 Cyg and TU Mus.

\begin{tabular}{lcccccc}
\hline Stars & $P$ & $\begin{array}{c}\dot{\tau} \\
(\mathrm{d} \mathrm{yr}\end{array}$ & $\begin{array}{c}\tau_{P} \\
(\mathrm{yr})\end{array}$ & $\begin{array}{c}\tau_{\mathrm{Th}} \\
(\mathrm{yr})\end{array}$ & $\begin{array}{c}\tau_{\mathrm{N}} \\
(\mathrm{yr})\end{array}$ & $\begin{array}{c}\dot{M} \\
\left(\mathrm{M}_{\odot} \mathrm{yr}^{-1}\right)\end{array}$ \\
\hline V382 Cyg & 1.8855 & $4.4 \times 10^{-7}$ & $4.3 \times 10^{6}$ & $9.2 \times 10^{3}$ & $8.5 \times 10^{6}$ & $4.3 \times 10^{-7}$ \\
TU Mus & 1.3873 & $4.0 \times 10^{-7}$ & $3.5 \times 10^{6}$ & $2.4 \times 10^{4}$ & $4.8 \times 10^{6}$ & $4.2 \times 10^{-7}$ \\
\hline
\end{tabular}

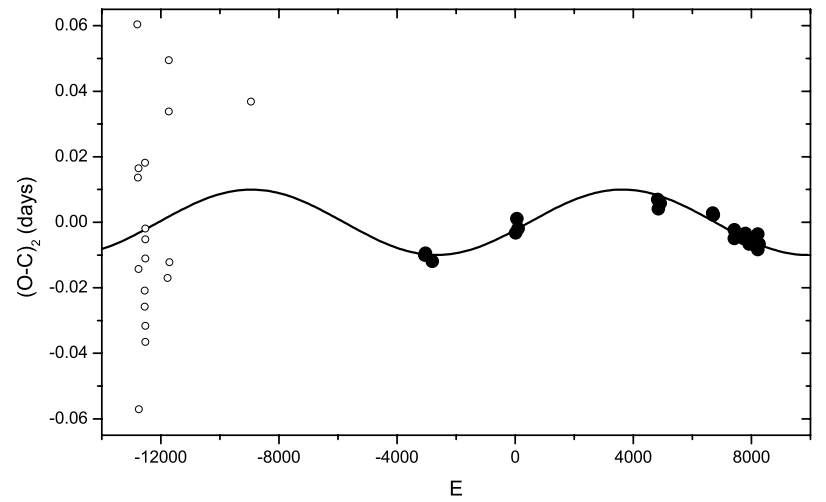

Figure 4. The same as Fig. 2, but for TU Mus.

where $M_{2}, R_{2}$ and $L_{2}$ are the masses, the radii and the luminosity of the less massive components. With the physical parameters listed in Table 1, the thermal and nuclear time-scales of the secondary stars can be calculated with equations (8) and (9). The results are listed in Table 6. As shown in the table, the time-scales of the orbital period changes are much longer than the thermal time-scales, but are close to the nuclear times-scales of the secondary components of both systems. This suggests that the long-term period changes can be interpreted by mass transfers on the nuclear time-scales of less massive components. By considering conservative mass transfer, a computation with the well-known equation,

$\frac{\dot{P}}{P}=3 \dot{M}\left(\frac{1}{M_{2}}-\frac{1}{M_{1}}\right)$,

leads to mass transfers at rates of $\mathrm{d} M / \mathrm{d} t=4.3 \times 10^{-7}$ and $4.2 \times$ $10^{-7} \mathrm{M}_{\odot} \mathrm{yr}^{-1}$ for V382 Cyg and TU Mus, respectively.

\subsection{The presence of unseen tertiary components}

The spectral types of V382 Cyg and TU Mus are O7.3V+O7.7V and $07.5 \mathrm{~V}+\mathrm{O} 9.5 \mathrm{~V}$, respectively. They are composed of two earlytype stars that presumably contain a convective core and a radiative envelope. This suggests that the period oscillations cannot be explained by the magnetic activity cycle mechanism, which is usually proposed to interpret the cyclic period changes of solar-type binary stars (e.g. Applegate 1992; Lanza, Rodonò \& Rosner 1998). Moreover, as shown in Figs 2 and 3, both the primary and the secondary times of light minimum follow the same general trends of the $\mathrm{O}$ $\mathrm{C}$ variations, which indicate that the period oscillations of the two systems cannot be caused by apsidal motion via eccentric orbits of the two binary stars. Therefore, a plausible explanation of the periodic changes of the orbital periods is the light-travel time effect via the presence of a tertiary component (e.g. Borkovits \& Hegedüs 1996).

Since the photoelectric and CCD data of the two systems do not cover a whole cycle, information on the orbital eccentricity of the third body is not clear. By considering that the third body is moving in a circular orbit, the projected radii of the orbits $a_{12}^{\prime} \sin i^{\prime}$ of the eclipsing pairs rotating around the mass central of the triple systems were computed with the equation

$a_{12}^{\prime} \sin i^{\prime}=A_{3} \times c$,

where $A_{3}$ is the amplitude of the $O-C$ oscillation and $c$ is the speed of light. The results are $a_{12}^{\prime} \sin i^{\prime}=2.56$ and 1.73 . Then, by using the parameters listed in Table 1, a computation with the equation

$f(m)=\frac{4 \pi^{2}}{G P_{3}^{2}} \times\left(a_{12}^{\prime} \sin i^{\prime}\right)^{3}$

leads to mass functions of $f(m)=0.0074$ and $0.0023 \mathrm{M}_{\odot}$ for $\mathrm{V} 382$ Cyg and TU Mus, respectively. $G$ and $P_{3}$ in equation (12) are the gravitational constant and the periods of the $O-C$ oscillations. Finally, the values of the masses and the orbital radii of the third components for different values of $i^{\prime}$ were estimated by the use of the following equation,

$f(m)=\frac{\left(M_{3} \sin i^{\prime}\right)^{3}}{\left(M_{1}+M_{2}+M_{3}\right)^{2}}$.

The relations between the orbital inclinations and the masses of the third bodies are plotted in Fig. 5. Those shown in Fig. 6 are the relations between the orbital inclinations and the orbital radii of the tertiary components in V382 Cyg and TU Mus. As displayed in Figs 5 and 6, the properties of the tertiary components in both the systems are nearly the same.

\section{DISCUSSIONS AND CONCLUSIONS}

\subsection{Tertiary invisible components quadruple systems}

Cyclic period oscillations appear to have been discovered in the orbital periods of V382 Cyg and TU Mus, and which undergo longterm continuous increases. The periodic changes in the orbital periods of both systems suggest that they contain third components. The

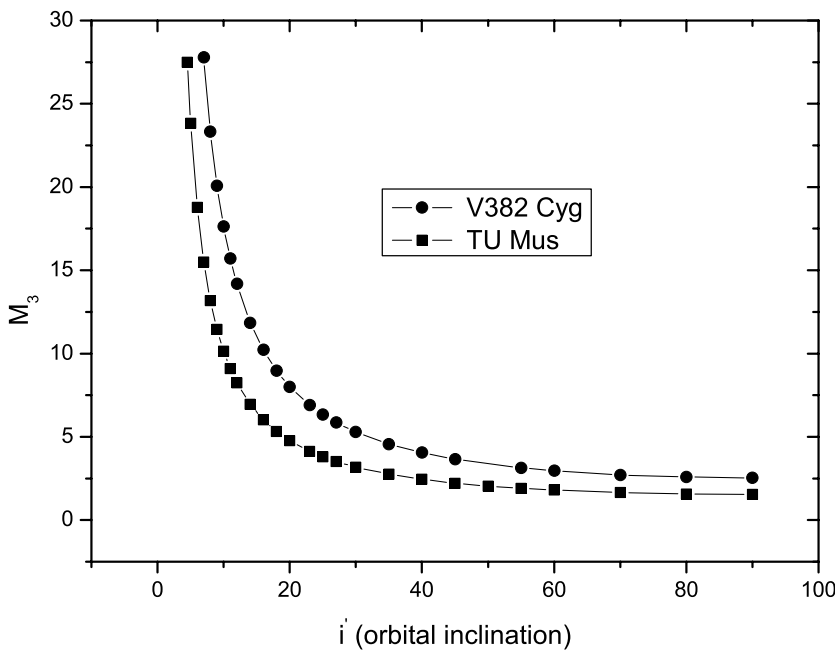

Figure 5. The relations between the masses of the third bodies $M_{3}$ and their orbital inclinations $i^{\prime}$ for two short-period O-type contact binaries V382 Cyg and TU Mus. 


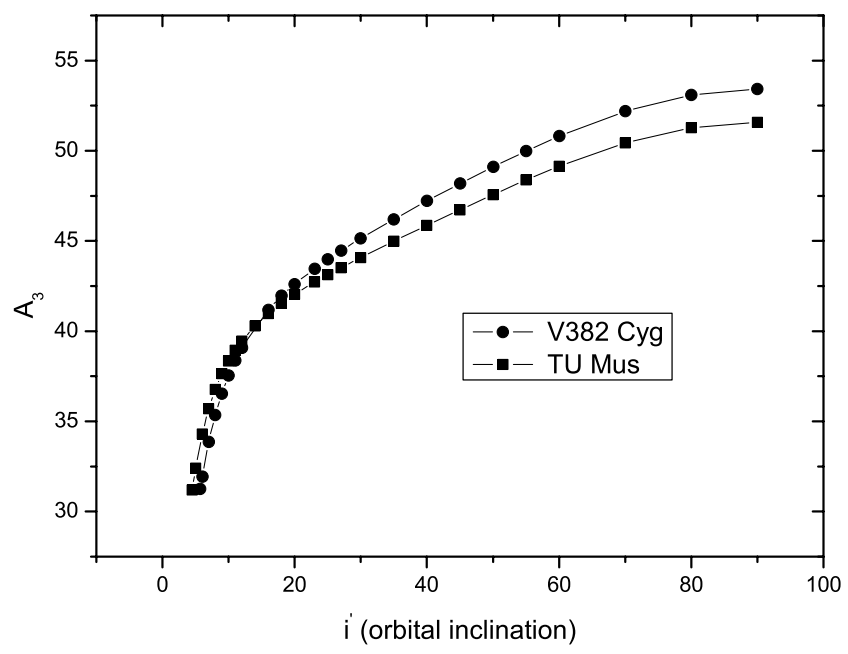

Figure 6. The relations between the orbital radii of the third bodies $A_{3}$ and their orbital inclinations $i^{\prime}$ for V382 Cyg and TU Mus.

lowest masses of the tertiary components in V382 Cyg and TU Mus are 2.56 and $1.55 \mathrm{M}_{\odot}$, respectively, which are much smaller than those of the central eclipsing pairs $\left(26.6+18\right.$ and $\left.23.5+15.3 \mathrm{M}_{\odot}\right)$. This indicates that the tertiary components are invisible because their luminosity is very small when compared with the central massive eclipsing pairs. It has been reported that there is a visual companion around TU Mus that is about 16 arcsec southeast of TU Mus. However, the largest orbital radius of the third body in TU Mus is only $51.6 \mathrm{au}$. This indicates that the tertiary component revealed by light-travel times effect is not the visual companion. The unseen third body is much closer to TU Mus and it is unresolved. For V382 Cyg, its visual companion is about 11.4 arcsec away from the central eclipsing pair (Chambliss 1992). The largest orbital radius of the third body is only 53.4 au suggesting that the observed light-travel time effect is not caused by the visual companion. The tertiary component is unresolved as in the case of TU Mus. The presence of invisible tertiary components in V382 Cyg and TU Mus suggests that they are quadruple systems. The eclipsing pairs have periods of less than two days, the third invisible components have periods of $\sim 48 \mathrm{yr}$ and outside the triple systems are the visual companions that have much longer periods. As in the case of $\eta$ Ori, both are quadruple system of hierarchy 3 (Chambliss 1992).

Photometric and spectroscopic investigations of V382 Cyg and TU Mus (e.g. Deǧirmenci et al. 1999; Terrell et al. 2003) have indicated that their components are main-sequence stars in an overcontact configuration. Case A mass transfer should be responsible for the overcontact configurations of the two systems. On the other hand, theoretical studies have shown that massive overcontact binary stars can be formed via Case A mass-transfer evolution if the orbital periods of the initial detached systems are short enough (e.g. Sybesma 1985, 1986; Pols 1994). The presence of the additional components in V382 Cyg and TU Mus may play an important role for their formations and evolutions by removing angular momentum from the central system. This makes the eclipsing pairs to have a lower angular momentum and a shorter initial orbital period (e.g. $P<3$ d) (Sybesma 1985). In the latter case, the initially detached binaries can evolve into overcontact configuration via a Case A mass transfer during their main-sequence evolutionary stage (e.g. Sybesma 1985, 1986; Pols 1994).

\subsection{Evolutionary states of V382 Cyg and TU Mus - compared} with that of late-type overcontact binary stars

The formation of massive overcontact binary stars has been investigated theoretically by several investigators (e.g. Sybesma 1985, 1986; Pols 1994). By including convective core-overshooting and stellar wind mass loss during the evolution, Sybesma (1985) suggested that binaries with initial masses of $20+10 \mathrm{M}_{\odot}(q=0.5)$ and initial orbital period shorter than $3 \mathrm{~d}$ evolve into a short-lived overcontact configuration during the rapid stage of Case A mass transfer, while systems with higher initial mass ratios (e.g. $q=0.8$ and 0.9 ) and still shorter periods form more stable overcontact systems within the slow phase (i.e. close to the end of Case A mass transfer; Sybesma 1986). In the well-known collection, Hilditch \& Bell (1987) compared observational data of seven massive overcontact binaries with stellar evolutionary grids and confirmed that these binary systems appear to be in overcontact states after rapid Case A and during slow Case A mass-transfer phases. The long-term continuous period increase of V382 Cyg and TU Mus can be plausibly interpreted as the results of mass transfer from the secondary to the primary components. It is discovered that the time-scales of the period variations of both systems are much longer than the thermal time-scales of the secondary components, but are close to the nuclear time-scales. This suggests that they have undergone rapid stage of Case A mass transfer at the thermal time-scales of the secondary components, and they are now on the slow evolutionary phase of Case A mass transfer.

Based on the orbital period changes of late-type overcontact binary stars, Qian (2001a,b, 2003) proposed that the combination of thermal relaxation oscillation (TRO) (e.g. Flannery 1976; Lucy 1976; Robertson \& Eggleton 1977) and variable angular momentum loss (AML) via change in overcontact depth drives this type of stars to oscillate around a critical mass ratio. This evolutionary scenario assumed that the variation of the overcontact depth can cause the variation of magnetic activity, thus changing the AML rate, which has been discussed by Vilhu (1981) and Smith (1984). ROSAT and IUE observations have suggested that compared with the fast rotating single stars and short-period detached systems, the level of magnetic activity of W UMa-type stars has been reduced by the presence of the common convective envelope (CCE) (Hrivnak et al. 2001; Stepien, Schmitt \& Voges 2001). This may be observational evidence of the conclusion that the overcontact depth couples with magnetic activity. The deeper the CCE, the weaker is the magnetic activity. To avoid the braking stage of the TRO model, Rahunen (1981) performed an interesting artificial computation to see what loss rate is needed to keep a system in overcontact. His results showed that a critical AML rate $\left(\mathrm{d} \ln j / \mathrm{d} t \sim 2 \times 10^{-9} \mathrm{yr}^{-1}\right)$ can keep W UMa-type binaries in shallow overcontact configuration. A larger AML rate will cause a system to soon reach the $L_{2}$-surface, and then to form a single star, whereas if a somewhat smaller loss rate is assumed, the system will be immediately broken and cyclic oscillation between overcontact and semidetached configurations occurs. If the rate of AML is variable with the overcontact depth, the system will oscillate around a critical mass ratio. In that way, the question that why the periods of some W UMa-type stars are increasing, while those of the others are decreasing can be explained. Components of late-type overcontact binaries remain in good overcontact configuration. For the two O-type overcontact binaries, V382 Cyg and TU Mus, as the orbital period increase, the overcontact configurations of both systems will be broken, which supports the conclusions of Sybesma $(1985,1986)$ that they will go through a short-lived overcontact configuration during the evolutionary phases of Case A 


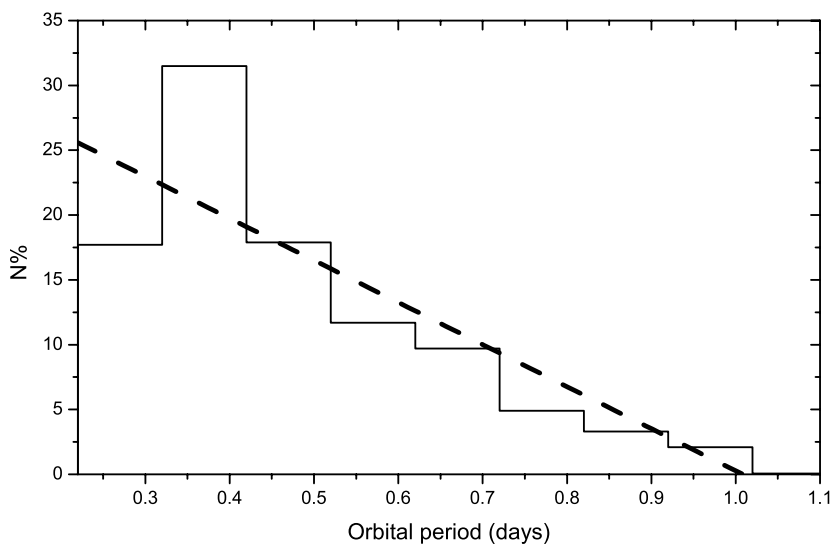

Figure 7. The distribution of overcontact binaries along with the orbital period. The dashed line is the general trend of this distribution. It is seen that the number of EW-type binaries stars is decreasing with increasing period.

mass transfer that is different from the situation of late-type contact binary stars.

From the periods of all EW-type binaries (usually overcontact systems) listed in the fourth edition of the general catalogue of variable stars (GCVS) (Kholopov et al. 1985, 1987), the distribution of overcontact binary stars along with the period is formed which is shown in Fig. 7 where the dashed line is the general trend of the distribution. As displayed in this figure, the number of overcontact binaries decreases with increasing period. It is well known that there is a tight relation between the spectral type and the period of overcontact binary stars, i.e. the longer the period the earlier is spectral type (Eggen 1961, 1967). This relation suggests that the number of late-type overcontact binaries is much higher than that of earlytype systems. This is potentially strong observational evidence for the conclusion that massive overcontact binaries are going through a short-lived overcontact configuration during the evolutionary phases of Case A mass transfer, whereas components of late-type overcontact binary remain in good overcontact configuration, driven by a combination of TRO and variable AML via change in overcontact depth.

\section{ACKNOWLEDGMENTS}

This work is partly supported by Yunnan Natural Science Foundation (No. 2005A0059M) and Chinese Natural Science (No. 10573032, No. 10573013 and No. 10433030). CCD photometric observations of V382 Cyg were obtained with the 1.0-m telescope at Yunnan Observatory in China and those of TU Mus with the 0.8-m telescope at the La Plata observatory in Argentina. QSB wishes to express his appreciation for the hospitality of the staff of Mt Suhora Observatory during his visit in Poland. We would like to thank the referee for those useful suggestions and comments which helped to improve the original manuscript.
Applegate J. H., 1992, ApJ, 385, 621

Borkovits T., Hegedüs T., 1996, A\&AS, 120, 63

Cester B., Fedel B., Giuricin G., Mardirossian F., Mezzetti M., 1978, A\&AS, 33,91

Chambliss C. R., 1992, PASP, 104, 663

Değirmenci Ö. L., Sezer C., Demircan O., Erdem A., Özdemir S., Ak H., Albayrak B., 1999, A\&AS, 134, 327

Devinney E. J., Twigg L. W., 1974, BAAS, 6, 335

Eggen O. J., 1961, R. Obs. Bull. No 31

Eggen O. J., 1967, Mem. R. Astron. Soc., 70, 111

Harries T. J., Hilditch R. W., Hill G., 1997, MNRAS, 285, 277

Hrivnak B. J., Guinan E. F., DeWarf L. E., Ribas I., 2001, AJ, 121, 1084

Flannery B. P., 1976, ApJ, 205, 217

Hilditch R. W., Bell S. A., 1987, MNRAS, 229, 529

Kholopov P. N. et al., 1985, GCVS, 4th edition. Nauka, Moscow Kholopov P. N. et al., 1987, GCVS, 4th edition. Nauka, Moscow Knipe G. F. G., 1971, Republ. Obs. Johannesb. Circ., 8, 8

Koch R. H., Siah M. J., Fanelli M. N., 1979, PASP, 91, 474

Kreiner M. J., Kim C.-H., Nha I.-S., 2001, An Atlas of O-C Diagrams of Eclipsing Binaries. Wydawnictwo Naukowe Akademii Pedagogicznej, Krakóv

Landolt A. U., 1964, ApJ, 140, 1494

Landolt A. U., 1975, PASP, 87, 409

Lanza A. F., Rodonò M., Rosner R., 1998, MNRAS, 296, 893

Lucy L. B., 1976, ApJ, 205, 217

Mayer P., 1980, Bull. Astron. Inst. Czechoslovakia, 31, 292

Mayer P., 2004, Bull. Astron. Inst. Netherlands, 5498

Mayer P., Wolf M., Muminovic M., Stupar M., 1986, IBVS 2942

Mayer P., Niarchos P. G., Lorenz R., Wolf M., Christie G., 1998, A\&AS, 130,311

Morgentoth O., 1935, Astron. Nachr., 255, 425

Oosterhoff P. Th., 1928, BAN, 4, 188

Oosterhoff P. Th., 1930, BAN, 5, 156

Pearce J. A., 1952, PASP, 64, 219

Petrov A. A., 1946, Perem. Zvezdy, 6, 72

Pols O. R., 1994, A\&A, 290, 119

Popper D. M., 1978, ApJ, 220, L11

Popper D. M., 1980, ARA\&A, 18, 115

Popper D. M., Hill G., 1991, AJ, 101, 600

Pojmanski G., 2002, Acta Astron., 52, 397

Qian S.-B., 2001a, MNRAS, 328, 635

Qian S.-B., 2001b, MNRAS, 328, 914

Qian S.-B., 2003, MNRAS, 342, 1260

Rahunen T., 1981, A\&A, 102, 82

Robertson J. A., Eggleton P. P., 1977, MNRAS, 179, 359

Smith R. C., 1984, QJRAS, 25, 405

Stepien K., Schmitt J. H. M. M., Voges W., 2001, A\&A, 370, 157

Stickland D. J., Lloyd C., Koch R. H., Pachoulakis I., 1995, Observatory, 115,317

Sybesma C. H. B., 1985, A\&A, 142, 171

Sybesma C. H. B., 1986, A\&A, 159, 108

Terrell D., Munari U., Zwitter T., Helson R. H., 2003, AJ, 126, 2988

Vilhu O., 1981, Ap\&SS, 78, 401

Whitney B. S., 1959, AJ, 64, 258

Wilson R. E., Rafert J. B., 1981, Ap\&SS, 76, 23

This paper has been typeset from a $\mathrm{T}_{\mathrm{E}} \mathrm{X} / \mathrm{L} \mathrm{T}_{\mathrm{E}} \mathrm{X}$ file prepared by the author. 